\title{
EVOLUTIONARY APPROACH TO THE SETI PROBLEM
}

\author{
V.V. Burdyuzha \\ Space Research Institute Academy of Sciences, USSR
}

V.I. Maron

Moscow Gubkin Oil and Gas Institute, USSR

M.D. Nussinov

125195 Moscow, Belomorskaja Str.22, Build.3, Apt. 351, USSR

Paper Presented by V. V. Burdyuzha

\begin{abstract}
Summary
We examined the substance's evolution impulse which led to the origin of life of the Earth by the example of the chain of self-connected molecular objects such as "...-molecule-macro-molecule (polymer)-biopolymer (RNA and DNA in genomes of living organisms beginning from unicellular microorganisms till Homo Sapiens) (M.D. Nussinov et al., JBIS, $\underline{38}$, NII,494 (1985). These molecular objects as the main formshaping factor appeared in Metagalaxy successively in time. The evolution of above objects had a self-organizational character which is proved by the decrease of informational entropy at the same length of informational "text" ("programme") or by decrease of number of the informational's "text alpha-bet letter". The last one is the most important regularity of substance's selforganization in Shennon understanding of such a process. The further evolution of molecular objects shall be created by Homo Sapiens intelligent as the "genomes" of self-organizing technologies.

The above mentioned molecular branch of substance's evolution passed in both connection and interrelation with simultaneously passed there evolution of the chain of astrophysical (conditional atomic) systems for which the main formshaping factor is already the gravitational interactions.

We estimated through an electromagnetic impulse area the characteristic time of impulse existence as equal to $\tau \sim(1$ to 2$) \cdot 10^{16} \mathrm{~s}$. Hence one can get the maximal characteristic linear size of the region of Metagalaxy which provides both substance and energy for supplying the self-organization of molecular systems. As it coincides roughly with the linear size of supercluster of galaxies ( $R$ $100 \mathrm{Mpc})$ and proves to be much less then Hubble radius $\left(\mathrm{R}_{\mathrm{H}}-2 \times 10^{28} \mathrm{~cm}\right)$. It suppose the nonuniqueness of similar impulses in our Metagalaxy. The rough minimal estimation of the number of such impulses (that would mean also the number of extraterrestrial intelligence) gives their value about $10^{5}$.
\end{abstract}

